\title{
Hydrocele and Unexpected Diagnosis: Malignant Mesothelioma of Tunica Vaginalis Testis
}

\author{
Samir Abdullazade ${ }^{\mathrm{a}}$, Ali Ergen ${ }^{\mathrm{b}}$, Dilek Ertoy Baydarª,
}

\begin{abstract}
Testicular mesothelioma originating from tunica vaginalis is a very rare disease. A 70-year-old man complained of left testicular swelling and was operated with the clinical diagnosis of hydrocele. Intra-operative examination revealed diffuse thickening of tunica vaginalis, which was thought to be inflammatory and benign by the urologist. Nevertheless an incisional biopsy was performed and atypical mesothelial proliferation was reported in histopathology. Morphologic findings were insufficient for definitive diagnosis of malignancy. Patient was followed-up for ten months. Due to the persistance of paratesticular thickening and additional appearance of nodularities, left inguinal orchiectomy was performed. Microscopy revealed classical malignant mesothelioma originating from tunica vaginalis. Two years after the diagnosis, patient had metastases to the paraaortic lymph nodes. Malignant mesothelioma of the testis may represent with diagnostic difficulties both clinically and pathologically. In all cases where paratesticular tissues may deviate from normal, mesothelioma must enter into the differential diagnosis.
\end{abstract}

Keywords: Mesothelioma; Testis; Tunica vaginalis; Hydrocele

\section{Introduction}

Malignant mesothelioma is the malignancy originating from the mesothelial cells lining the pleural and peritoneal cavities, as well as the pericardium and tunica vaginalis [1]. It can be uni- or multifocal tumor, or may involve its whole membrane of origin in a diffuse manner. Mesothelioma of the tunica vaginalis of testis and inguinal canal is very rare. The non-specific symptoms, broad age distribution and lack of tumor markers make their pre-operative diagnosis challenging. Although ultrasound is considered as the imaging modality of choice in the evaluation of the scrotal tumors today, reports on ultrasound features of mesothelioma arising from tunica vaginalis are limited [2]. And indeed these neoplasms present considerable diagnostic difficulty not only for the clinician and radiologist but also for the pathologist when the material submitted to histology is small and superficial. Herein, we describe an additional case of malig-

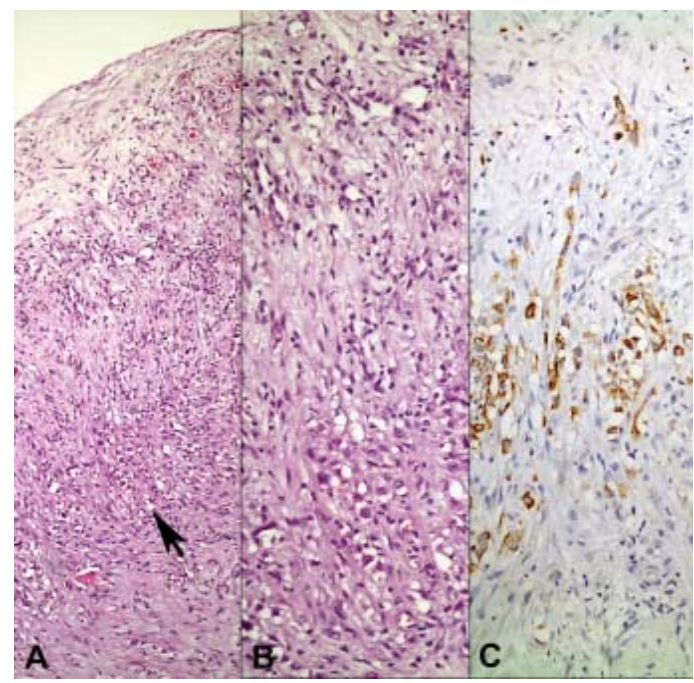

Figure 1. Biopsy from tunica vaginalis showing mesothelial cell proliferation (arrow). Panel $\mathrm{B}$ and $\mathrm{C}$ represent the area pointed by the arrow (A: H-E x 40; B: H-E x 200; C: Immunohistochemistry, anti-calretinin $\mathrm{Ab} \times 200)$. 


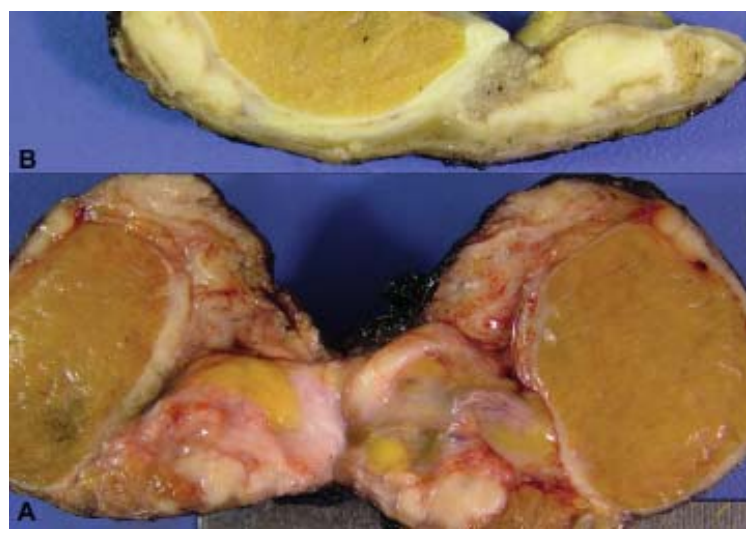

Figure 2. Macroscopical appearance of the tumor (A: Before fixation; B: After fixation).

nant mesothelioma derived from tunica vaginalis, which was incidental and caused significant trouble in diagnosis.

\section{Case Presentation}

A 70-year-old man presented with the complaint of swelling in the left testis. He was born in South-east part of Turkey. His past medical history was significant for type II diabetes mellitus, hypertension and colloidal goiter. Scrotal ultrasonography showed accumulation of fluid in scrotal sac. He underwent operation with the preliminary diagnosis of hydrocele. During the operation, diffuse mild thickening of tunica vaginalis was noted in addition to hydrocele. This was assigned to reactive inflammatory process secondary to hydrocele by the surgeon, but still a small incisional biopsy was performed. Pathology showed prominent proliferation

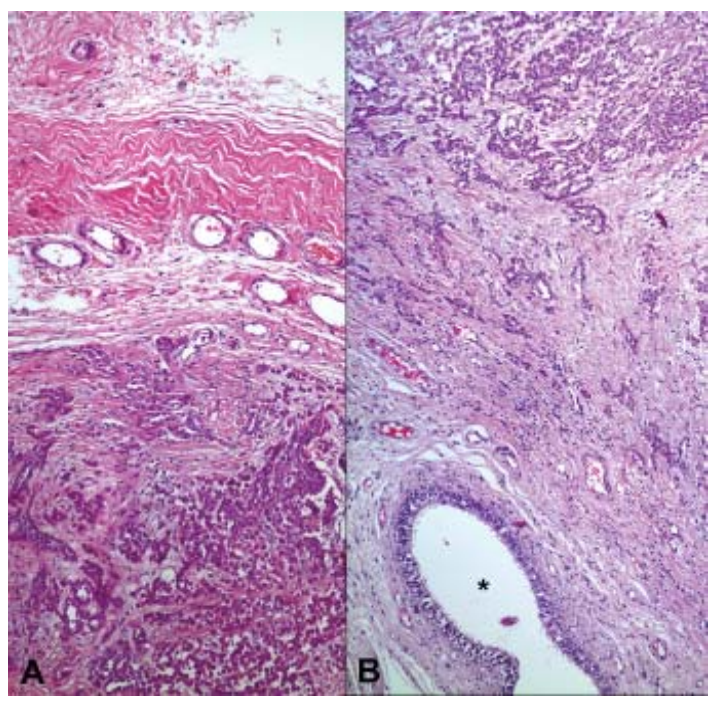

Figure 3. Tumor infiltrating paratesticular tissues. *shows an epidydimal ductus (A: H-E x 100; B: H-E x 40).

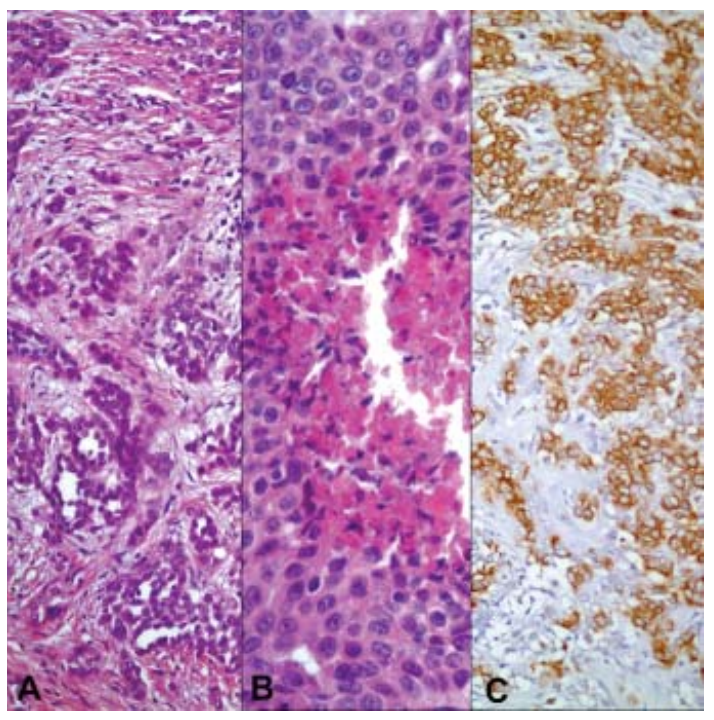

Figure 4. Neoplasm showing tubular (panel A) and solid patterns with necrosis (panel $B$ ). Neoplastic cells diffusely express a mesothelial marker, HBME-1 (panel C) (A: H-E x 200; B: H-E x 400; C: Immunohistochemistry, anti- HBME-1 Ab x 200).

of mesothelial cells (Fig. 1). There was a suspicion of malignancy, but findings were not found satisfactory for definitive diagnosis of mesothelioma. The nuclear pleomorphism was mild and no necrosis was identified. Because of the small size of biopsy, the depth of the lesion and the presence of invasion could not be determined. Since the reactive mesothelial proliferation could not be excluded with certainty, it was decided to follow the patient instead of

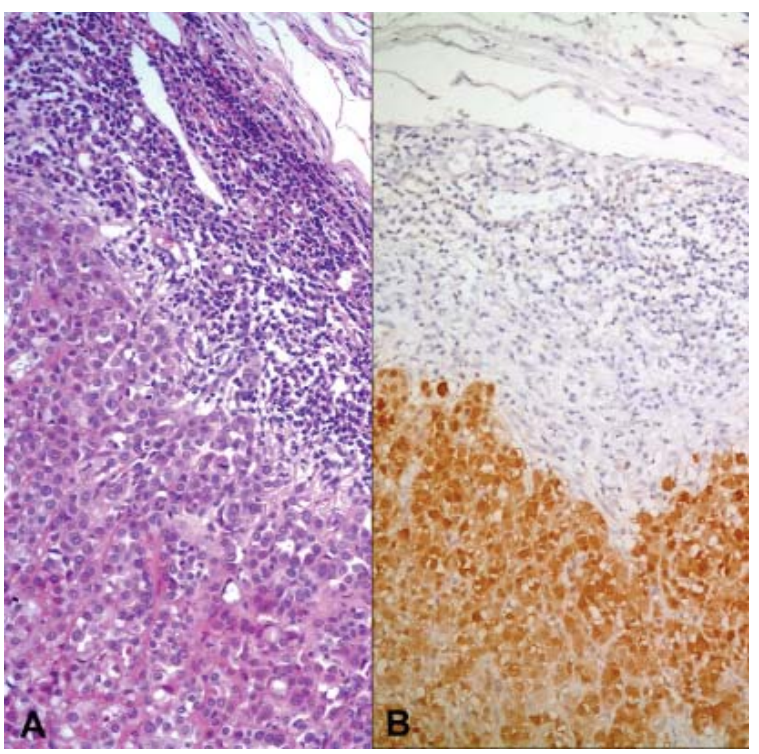

Figure 5. One of the lymph nodes with metastasis of mesothelioma (A: H-E x 200; B: Immunohistochemistry, anticalretinin $A b \times 200)$. 
radical surgery. However, after ten months no remission but instead emergence of nodular lesions in tunica vaginalis was observed. Then left inguinal orchiectomy was undertaken.

Macroscopical examination showed encasement of testis by rigid and diffusely thick solid testicular tunica, mainly at posterior and lateral sides. There were also irregular nodulations in addition to widespread thickening. The diameter of nodules varied from $0.5 \mathrm{~cm}$ to $2.5 \mathrm{~cm}$ (Fig. 2).

Microscopic examination revealed classical malignant mesothelioma in the epithelial form mainly with tubulopapillary and solid patterns, but nearly $10 \%$ sarcomatous component was also present (Fig. 3, 4). Nodules contained central necrotic areas. Large concentric calcifications within dens fibrosis were frequent. Neoplastic cells had oval to round vesicular nuclei, prominent nucleoli and moderately abundant eosinophilic cytoplasm. They stained immunohistochemically with mesothelial markers calretinin, HBME-1, thrombomodulin and cytokeratin 5/6 in addition to GLUT1 (Fig. 4). CD117, OCT3/4 and inhibin were negative. Tumor invaded paratesticular soft tissues, epidydimis, root of spermatic cord and tunica albuginea. There was no testicular parenchymal involvement. Spermatic cord margin and all surgical borders were free of tumor.

Systemic imaging studies did not reveal lymph nodal or visceral metastases. He was not given adjuvant therapy. But after 10 months follow-up, CT scans showed enlarged intraabdominal lymph nodes. Paraaortic lymphadenectomy was performed. Four lymph nodes out of 23 had metastatic mesothelioma (Fig. 5). The greatest diameter of the largest metastatic lymph node was $1.5 \mathrm{~cm}$ with the tumor showing extranodal extension. Patient was given salvage chemotherapy. Eighteen months after lymphadenectomy, he is alive without additional recurrences.

\section{Discussion}

Diagnosis of mesothelioma especially in tunica vajinalis is a difficult task. Rarity of the lesion makes it more challenging. It was first described by Barbera and Rubino in 1957 [3]. Since then, only about 100 cases have been reported in the literature [4].

Patients usually present with hydrocele and/or palpable mass in scrotum. Macroscopically, tumor involves the tunica vaginalis either diffusely or in a multinodular manner, with invasion into the surrounding structures. Scrotal space may contain multifocal, friable, papillary excrescences. The list for clinical differential diagnosis in the cases where a mass is detected prior to surgery is long and it may include rete neoplasms, ovarian surface epithelial type tumors, metastatic carcinomas, paratesticular sarcomas and even germ cell tumors when there is testicular parenchymal invasion. The same differential is also valid for the pathological evaluation. Once the proper diagnosis is considered, immunohistochemistry can resolve the problem easily. Mesothelial cells express cytokeratin 5/6, epithelial membrane antigen, calretinin, vimentine, HBME1 , thrombomodulin. On the contrary to many epithelial tumors, they are negative for Leu M1, Ber-EP4 and CEA. However, the histological diagnosis can be hard when there is only a superficial biopsy to evaluate. As in our case, the determination of the stromal invasion and differentiation of the lesion from reactive mesothelial proliferations can be problematic in small tissue samples. Our patient presented with hydrocele with mild tunical thickening noted at the operating table, but without a distinct mass. Therefore, inflammatory changes and hyperplastic mesothelial cell reactions were the main preliminary considerations. With more attention directed to the life of the patient, we noted that he was exposed to the asbestos during his childhood. His birth place was the rural area of the country where the pleural mesothelioma has high incidence. However, even with this information, we were indeterminate to sign out the biopsy as malignant. The proliferating mesothelial cells looked atypical with large nuclei and prominent nuclei, but necrosis or mitosis could not be identified. More importantly, it was not possible to determine the depth of lesion and the presence of actual invasion. So, the certain diagnosis was delayed until radical orchiectomy was performed 10 months later due to new development of nodular lesions in the tunica vaginalis.

The only established predisposing factor for paratesticular malignant mesothelioma is the asbestos exposure. The certain regions in Turkey have the world's highest prevalence of malignant pleural mesothelioma. Environmental exposure to asbestos and erionite (a naturally occurring non-asbestos but asbestos-like fibrous mineral that belongs to a group called zeolites) is thought to cause the disease. Our patient was born in the southeast part of Turkey where people living are exposed to the soil containing serpentine and amphibole asbestos fibers. This soil is quarried from mountains by men and used as a whitewash coating for the walls and floors of the houses, a procedure repeated every year. Thus, householders are continually exposed to asbestos fibers starting from birth [5, 6]. Although it has been reported in a 6-year-old child, more than two-thirds of the patients with paratesticular mesotheliomas are older than 45 with a median age of 60 [7] and our patient was 70. There is generally a long latent period after the first asbestos exposure to develop the disease.

Malignant mesothelioma of tunica vaginalis is a highly aggressive disease. Despite relatively bland cytologic features in many of these tumors, they have well-documented metastatic potential. Radical inguinal orchiectomy is the treatment of choice in the patients with localized disease. The utility of lymph node dissection, and adjuvant chemotherapy and/or radiotherapy has not been clarified. Metastasis occurs early through the lymphatics mostly to para-aortic, less 
frequently inguinal, iliac or supraclavicular nodes, and has been reported in 14.9-31\% of the cases [7, 8]. Visceral spread is rare, but can occur to the organs such as liver and lungs. The main prognostic factor is the extent of disease at the time of presentation. When distant metastases are present, the prognosis is dismal where the survival is usually not more than few months. Organ confined tumors, especially in younger ages before 60 achieve better natural history [7, 9].

The diagnosis of paratesticular malignant mesothelioma can be difficult, both from a clinical and pathological point of view. Awareness and proper recognition of this entity are essential to reach the correct and prompt diagnosis. High level of suspicion is required whenever there is an unexplained or even a reactive looking alteration of tunica vaginalis. The life of the patient must be carefully explored in regard to environmental or occupational asbestos exposure, which can be a good support for the right way of approach.

\section{Conflicts of Interest}

There is no conflict of interest for disclosure.

\section{References}

1. Jett J, Aubry M. Malignant pleural mesothelioma. In Clinical Respiratory Medicine. Edited by Albert, Spiro,
Jett. 2nd edition; Philadelphia. Mosby, 2004. Pages 735741.

2. Mak CW, Cheng TC, Chuang SS, Wu RH, Chou CK, Chang JM. Malignant mesothelioma of the tunica vaginalis testis. Br J Radiol. 2004;77(921):780-781.

3. Barbera V, Rubino M. Papillary mesothelioma of the tunica vaginalis. Cancer. 1957;10(1):183-189.

4. Chen JL, Hsu YH. Malignant mesothelioma of the tunica vaginalis testis: a case report and literature review. Kaohsiung J Med Sci. 2009;25(2):77-81.

5. Yazicioglu S. Pleural calcification associated with exposure to chrysotile asbestos in southeast Turkey. Chest. 1976;70(1):43-47.

6. Yazicioglu S, Ilcayto R, Balci K, Sayli BS, Yorulmaz B. Pleural calcification, pleural mesotheliomas, and bronchial cancers caused by tremolite dust. Thorax. 1980;35(8):564-569.

7. Plas E, Riedl CR, Pfluger H. Malignant mesothelioma of the tunica vaginalis testis: review of the literature and assessment of prognostic parameters. Cancer. 1998;83(12):2437-2446.

8. Yamanishi T, Wakisaka M, Ito H, Matsuzaki O, Nagao K, Shimazaki J. Malignant mesothelioma of the tunica vaginalis testis. Eur Urol. 1984;10(3):207-209.

9. Gupta NP, Agrawal AK, Sood S, Hemal AK, Nair M. Malignant mesothelioma of the tunica vaginalis testis: a report of two cases and review of literature. J Surg Oncol. 1999;70(4):251-254. 